Artículo original

(Original paper)

\title{
VARIACIÓN ESTACIONAL DEL CONTENIDO NUTRICIONAL DE LA DIETA DEL BORREGO CIMARRÓN DEL DESIERTO (OVIS CANADENSIS WEEMSI), EN BAJA CALIFORNIA SUR, MÉXICO
}

\author{
SEASONAL VARIATION OF THE NUTRITIONAL CONTENT IN THE DIET OF THE \\ DESERT BIGHORN SHEEP (OVIS CANADENSIS WEEMSI), IN BAJA CALIFORNIA SUR, \\ MEXICO
}

\author{
ISRAEL GUERRERO-CÁRDENAS ${ }^{1,2}$, SERGIO ÁLVAREZ-CÁRDENAS ${ }^{2}$, SONIA GALLINA ${ }^{3 *}$, PABLO \\ CORCUERA $^{4}$, RAFAEL RAMÍREZ-ORDUÑ̃ ${ }^{5}$, IVONNE TOVAR-ZAMORA ${ }^{2}$ \\ ${ }^{1}$ Universidad Autónoma Metropolitana-Xochimilco. Doctorado en Ciencias Biológicas y de la Salud. Calzada del Hueso 1100 , \\ Coyoacán 04960. Ciudad de México, México. <guerrero04@cibnor.mx> \\ ${ }^{2}$ Centro de Investigaciones Biológicas del Noroeste, S. C. Instituto Politécnico Nacional 195, Col. Playa Palo de Santa Rita Sur \\ 23090, La Paz, Baja California Sur, México. <salvarez04@cibnor.mx>; <itovar@cibnor.mx> \\ ${ }^{3}$ Instituto de Ecología A. C. Red de Biología y Conservación de Vertebrados. Carretera Antigua a Coatepec 351, El Haya, Xalapa, \\ Veracruz, C.P.91070. México. <sonia.gallina@inecol.mx> \\ ${ }^{4}$ Universidad Autónoma Metropolitana-Iztapalapa. Departamento de Biología, Área Ecología. Av. San Rafael Atlixco 186, \\ Iztapalapa 09340. Ciudad de México, México. <pcmr@xanum.uam.mx> \\ ${ }^{5}$ Laboratorio de Nutrición y Morfofisiología Animal, Universidad Autónoma de Baja California Sur, Km 5.5 Carretera al Sur, \\ 23088. La Paz, Baja California Sur, México. <rramirez@uabcs.mx> \\ *Autor de correspondencia: <sonia.gallina@inecol.mx>
}

Recibido: 22/11/2017; aceptado: 24/05/2018; publicado en línea: 25/10/2018

Editor responsable: Vinicio Sosa

Guerrero-Cárdenas, I., Álvarez-Cárdenas, S., Gallina, S., Corcuera, P., Ramírez-Orduña, R., Tovar-Zamora, I. (2018) Variación estacional del contenido nutricional de la dieta del borrego cimarrón (Ovis canadensis weemsi), en Baja California Sur, México. Acta Zoológica Mexicana (nueva serie), 34, 1-18. DOI: https://doi.org/10.21829/azm.2018.3412113

RESUMEN. El borrego cimarrón (Ovis canadensis) es uno de los grandes ungulados silvestres nativos de Norte América. Es un herbívoro selectivo, que se alimenta de forrajes con valores altos en nutrientes. El objetivo de este estudio fue evaluar la variación estacional en la calidad nutricional de las especies consumidas por el borrego cimarrón en la Sierra El Mechudo, BCS México. Las muestras se recolectaron en las cuatro estaciones de los años 2010 y 2011, y para los análisis consideramos porcentajes de ceniza, proteína cruda (PC), fibra detergente neutra (FDN), fibra detergente ácida (FDA), extracto etéreo (EE) y digestibilidad in vitro de la materia seca (DIVMS). Se encontraron cambios estacionales en el contenido de nutrientes de las especies de plantas que se estudiaron. El mayor número de forrajes con altos porcentajes de nutrientes se encontró durante la primavera en Viscainoa geniculata (71.9\% DIVMS), Buddleja corrugata (11.7\% ceniza), Lysiloma candida (22.03\% proteína cruda), Condalia globosa (56.9\% FDA), Aristida adscensionis (80.2\% FDN) y Bursera epinnata (5.3\% EE). En invierno, los porcentajes más elevados de nutrientes fueron para Opuntia cholla (71.7\% IVDMD), Fouquieria diguetii (16.04\% PC), Bouteloua aristidoides (78.4\% NDF) y Croton caboencis (5.7\% EE). Mientras que para el verano y el otoño solo se encontraron dos especies con altos valores: L. candida (9.1\% ceniza) y Krameria parvifolia (50.2\% FDA). Con base en los dos indicadores de selección (Bonferroni e Ivlev) tres especies por año resultaron ser principalmente seleccionadas durante las diferentes estaciones. Caesalpinia placida fue la única especie seleccionada en los dos años, particularmente durante otoño e invierno. Encontramos 
que los principales nutrientes (ceniza o minerales, proteína cruda y extracto etéreo) del forraje consumido estuvieron asociados principalmente con la segunda etapa de la gestación (finales de verano y todo el otoño) y con la primera y segunda etapas del parto y lactancia (invierno y primavera). En la Sierra El Mechudo las hembras seleccionan especies de plantas de buena y mediana calidad, obteniendo de ellas la mejor combinación de nutrientes.

Palabras clave: Análisis químicos proximales; condición fisiológica; energía; extracto etéreo; DIVMS; fibras; proteína cruda

Guerrero-Cárdenas, I., Álvarez-Cárdenas, S., Gallina, S., Corcuera, P., Ramírez-Orduña R., TovarZamora, I. (2018) Seasonal variation of the nutritional content in the diet of the desert bighorn sheep (Ovis canadensis weemsi), in Baja California Sur, Mexico. Acta Zoológica Mexicana (nueva serie), 34, 1-18. DOI: https://doi.org/10.21829/azm.2018.3412113

\begin{abstract}
Bighorn sheep (Ovis canadensis) is one of the largest native wild ungulates in North America. It is a selective herbivore, feeding on forage with high nutrient values. The aim of this study was to evaluate the seasonal variation in the nutritional quality of the species consumed by bighorn sheep in the Sierra El Mechudo, BCS Mexico. Samples were collected in the four seasons of 2010 and 2011, and for the analyses we consider percentages of ash, crude protein (CP), neutral detergent fiber (NDF), acid detergent fiber (ADF), ether extract (EE), and in vitro digestibility of dry matter (IVDMD). Seasonal changes were found in the nutrient content of plant species that were studied. The largest number of forages with high percentages of nutrients found in spring were for Viscainoa geniculata (71.9\% IVDMD), Buddleja corrugata (11.7\% ash), Lysiloma candida (22.03\% crude protein), Condalia globosa (56.9\% FDA), Aristida adscensionis (80.2\% NDF) and Bursera epinnata (5.3\% EE). In winter, the highest percentages of nutrients were for Opuntia cholla (71.7\% IVDMD), Fouquieria diguetii (16.04\% PC) Bouteloua aristidoides (78.4\% NDF) and Croton caboencis (5.7\% EE). While for the summer and fall only two species with high values were found: L. candida $(9.1 \%$ ash) and Krameria parvifolia (50.2\% FDA). Based on two selection indicators (Bonferroni and Ivlev) three species per year were found to be primarily selected during different seasons. Caesalpinia placida was the only species selected in the two years, particularly during autumn and winter. We found that the principal nutrients (ash or minerals, crude protein, ether extract) of forage consumed were associated mainly with the second stage of gestation (late summer and all autumn) and with the first and second stages of parturition and lactation (winter and spring). In the Sierra El Mechudo, females select plant species of good and medium quality, getting the best combination of nutrients.
\end{abstract}

Key words: Crude protein; energy; ethereal extract; fibers; IVDMD; physiological condition; proximal chemical analyses

\title{
INTRODUCCIÓN
}

Los rumiantes silvestres en las zonas áridas experimentan marcadas fluctuaciones en su alimentación y la calidad de ésta debido a la estacionalidad de este tipo de ambientes (Poppi \& McLennan, 1995). Los patrones estacionales de los nutrientes son fundamentales, ya que influyen fuertemente en el éxito reproductivo y la probabilidad de supervivencia de los animales (Cook et al., 2007). Específicamente, ungulados como el borrego cimarrón seleccionan plantas para su mantenimiento y reproducción, así como para enfrentar condiciones climáticas adversas y la depredación (Bailey et al., 1980). La alimentación (qué plantas consume) y la nutrición (contenido de nutrientes de las plantas consumidas) de estos ungulados silvestres son dos de los principales factores que regulan su distribución y abundancia en un área determinada (Tarango \& Krausman, 1997). 
El borrego cimarrón se encuentra particularmente adaptado a las condiciones áridas y montañosas de su área de distribución, debido a sus características conductuales y adaptaciones morfológicas y fisiológicas (Smith \& Krausman, 1988; Sandoval et al., 2014). Particularmente las subespecies de la Península de Baja California han tenido que adaptarse a condiciones extremas de aridez (Valdez \& Krausman, 1999), con prolongados períodos de sequía, y alimento de baja calidad (Sandoval et al., 2014). Dos de las principales adaptaciones de la especie para mejorar su alimentación son la longitud del tracto digestivo (permitiendo un mayor tiempo de paso del alimento) y el gran tamaño de los molares (lo que permite desde el inicio de la digestión una mejora para extraer nutrientes), permitiendo un mejor proceso digestivo, lo que se traduce en una optimización de los recursos alimenticios consumidos (GuerreroCárdenas et al., 2016).

Los factores del hábitat que más afectan el consumo o preferencia de los alimentos en ungulados silvestres son la disponibilidad, el contenido nutrimental (proteína, fibra, lignina, aceites esenciales, vitaminas, minerales, compuestos secundarios, etc.) y la digestibilidad (Ramírez-Lozano, 2004). El borrego cimarrón es uno de los grandes ungulados silvestres nativos de Norte América, y en México se encuentran tres de las siete subespecies que se distribuyen desde Canadá y USA: O. c. cremnobates en Baja California, O. c. weemsi en Baja California Sur y O. c. mexicana en Sonora (Sandoval et al., 2014).

En la Península de Baja California el borrego cimarrón se considera una especie bandera de los ecosistemas árido-montañosos (Escobar-Flores et al., 2016) sujeta a protección especial, de acuerdo con la NOM-059-SEMARNAT-2010 (SEMARNAT, 2010). Jiménez \& Hernández (2010) estiman que en Baja California Sur la población de borrego cimarrón se encuentra distribuida en tres áreas principales: las Tres Vírgenes (Santa Rosalía, en la parte norte del Estado), La Giganta (Loreto, en la porción media) y El Mechudo (La Paz, en la parte sur) que corresponden al 40\% de su rango original.

En los ambientes áridos y desérticos de Norte América, la falta de alimento de buena calidad, hace que los animales sufran estrés nutricional. Esto es más evidente durante la estación seca que es la época más estresante del año, y que es cuando los borregos silvestres se ven forzados a consumir especies vegetales que son ricas en nutrientes pero también lo son en compuestos secundarios, por lo que normalmente no son consumidas (Hoffman, 1989; Holt et al., 1992; Wagner \& Peek, 2006).

Investigaciones sobre la dieta de borrego cimarrón en diferentes áreas de Estados Unidos, particularmente con subespecies del desierto, sugieren que es un consumidor oportunista, principalmente de pastos (gramíneas) y herbáceas (Geist, 1971; Cunningham, 1989; DeYoung et al., 2000). Sin embargo, Guerrero-Cárdenas et al. (2016) encontraron que los borregos fueron muy selectivos durante los dos años del estudio en la Sierra el Mechudo, debido a que seleccionaron principalmente especies arbustivas y arbóreas, y en menor proporción, pastos y otras herbáceas. Este patrón no se presenta en otras partes de su distribución, aunque investigadores de la United States Fish and Wildlife Service (2008) encontraron que los borregos de California son altamente selectivos, pero ahí eligen particularmente pastos, y en menor porcentaje especies arbustivas ya que en ese sitio los pastos proporcionan más energía.

La preferencia del borrego cimarrón por las especies vegetales que consume de acuerdo a su disponibilidad y calidad, son importantes para facilitar la detección de las especies clave en su dieta (Hanley, 1997; Martínez, 2010). Estas preferencias pueden ser explicadas probando la hipótesis de la calidad selectiva (Corriale et al., 2011), la cual establece que las plantas seleccionadas presentan un valor nutrimental más alto, durante aquellas etapas fisiológicas donde los borregos requieren de mayor demanda de nutrientes. De acuerdo con lo anterior, el objetivo del presente estudio fue evaluar la variación estacional en la calidad nutricional de las especies vegetales que consumen los borregos y determinar si tal variación se relaciona con la etapa fisiológica por la que atraviesa el borrego. 


\section{MATERIALES Y MÉTODOS}

Área de estudio. El estudio se realizó durante dos años (2010 y 2011) en el extremo sur del macizo montañoso de la Sierra El Mechudo, al noroeste de la Bahía de la Paz, en Baja California Sur, México. Los muestreos se realizaron en un área de 117, 578 ha en la localidad del Junco, localizada en la zona de San Juan de la Costa, entre las coordenadas geográficas $24^{\circ} 24^{\prime} 44.17 \mathrm{~N}$ y $110^{\circ} 45^{\prime} 9.10$ O (Fig. 1). Este sitio, junto con las localidades del Camarón y el Cajete, se consideran como el límite sur de la distribución natural de los borregos de montaña de Norte América (Arriaga et al., 2002; Álvarez-Cárdenas et al., 2009).

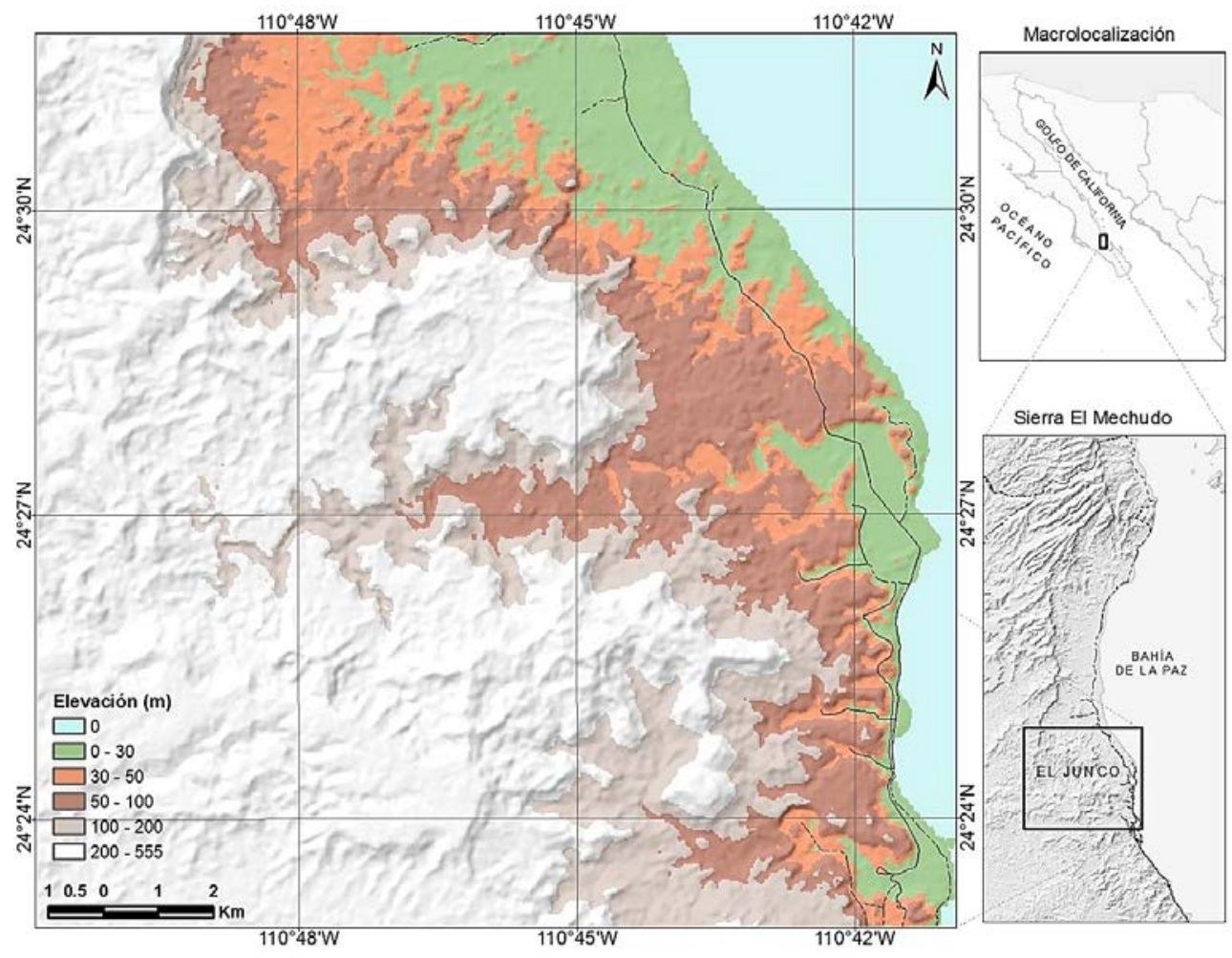

Figura 1. Localización de la zona del estudio, El Junco que pertenece al macizo montañoso de la Sierra El Mechudo, en la parte sur de Baja California Sur, México.

El rango de altitud de la localidad se encuentra entre los 100-400 m (7,700 ha), con algunas áreas en el rango de los 400-600 m (1,500 ha). El área presenta tierras por abajo de los $100 \mathrm{~m}$ y no hay sitios que alcancen más de los $600 \mathrm{~m}$. Existen sitios con terreno plano (800 ha), más del $80 \%$ del terreno corresponde a sierras bajas (3,600 ha) y a sierras medianas con cañadas (4,500 ha). Una pequeña superficie (1,100 ha) está conformada por sierras altas con cañones. Las laderas orientales son empinadas y orientadas hacia el Golfo de California, y están compuestas de cañones, precipicios y lechos secos de arroyos (Álvarez- Cárdenas et al., 2009).

En este sitio se encuentran tres tipos de vegetación: 1) matorral espinoso (especies arbóreas y arbustivas caducifolias con espinas prominentes) en las partes más altas, con especies como Pachycormus discolor, Stenocereus thurberi y Opuntia sp.; 2) matorral inerme (especies sin espinas y de hojas pequeñas) en laderas y cañones, dominado por Lysiloma candida, Ruellia californica y Fouquieria diguetii y 3) matorral inerme-cardonal (asociación de diferentes especies sin espinas y especies suculentas 
con espinas) en las partes más bajas, dominado por Pachycereus pringlei, Bursera epinnata y Prosopis glandulosa (Guerrero-Cárdenas et al., 2016).

El clima que se presenta en la zona es del tipo seco desértico cálido, con una temperatura media mensual que oscila entre 17.9 y $35.3^{\circ} \mathrm{C}$ en los meses de enero y agosto y una media anual que varía entre los 22 y $35^{\circ} \mathrm{C}$ (García, 1988). El promedio de precipitación anual es de $140 \mathrm{~mm}$ y se presenta en dos periodos: el más intenso en agosto-septiembre (incidencia de huracanes), seguido del de diciembre-enero (5 a 10.2\% del total anual) que corresponde a la lluvia invernal (SARH, 1983). En la zona se presentan periodos cíclicos de sequía que pueden durar varios años (Guerrero-Cárdenas et al., 2016). Durante el estudio, la temperatura media anual fue de $23.7^{\circ} \mathrm{C}$, la media máxima de $29.4^{\circ} \mathrm{C}$ y la media mínima de $18.0^{\circ} \mathrm{C}$. La temperatura máxima registrada fue de $45^{\circ} \mathrm{C}$ durante el verano de los dos años muestreados. El régimen de lluvias fue muy escaso, con un promedio de precipitación de $3.2 \mathrm{~mm}$ (Fig. 2).

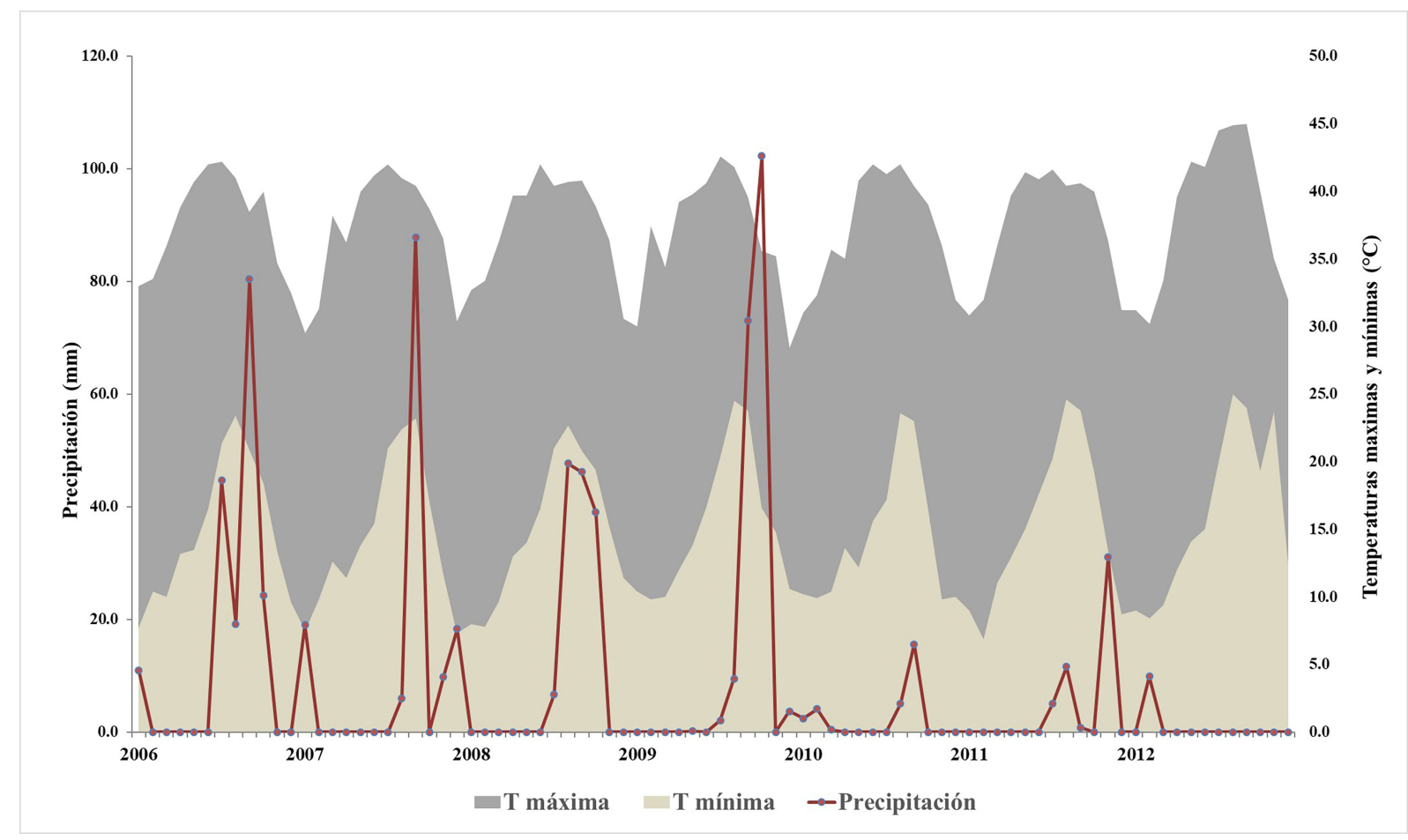

Figura 2. Climograma de temperaturas máximas, mínimas y precipitación, durante siete años (2006-2012) en el área de estudio. Solo 2010 y 2011, son los años en los que se colectó la información de campo.

El estudio se realizó en los años 2010 y 2011. Tomando en consideración las etapas fisiológicas para los borregos del desierto (Monson \& Sumner 1982; Valdez \& Krausman, 1999), el año se dividió en cuatro periodos:

1) Verano (julio-agosto-septiembre), reproducción y primera etapa de la gestación.

2) Otoño (octubre-noviembre-diciembre), segunda etapa de la gestación.

3) Invierno (enero-febrero-marzo), primera etapa de nacimientos y lactación.

4) Primavera (abril-mayo-junio), segunda etapa de nacimientos y lactación.

Muestreo de material vegetal. Previo a este estudio, mediante la técnica microhistológica para residuos vegetales en heces, Guerrero-Cárdenas et al. (2016) identificaron 34 especies vegetales en 2010 y 39 en 2011. En total, los borregos consumieron 47 especies que de manera porcentual correspondieron a $62.2 \%$ de arbustos, $26.9 \%$ de herbáceas, $10.6 \%$ de árboles y $0.2 \%$ de suculentas. 
Una vez establecida la dieta de los borregos, se colectaron las especies registradas durante los meses de abril-mayo (primavera), julio y septiembre (verano), octubre-noviembre (otoño) y enero-febrero (invierno), de los dos años. A partir de muestreos aleatorios (recorridos al azar en diferentes sitios del área de estudio), se colectaron ramas y hojas (no se encontraron frutos durante el estudio) en cada época. Estas fueron agrupadas por especie y forma de vida (árboles, arbustos, herbáceas y suculentas) y posteriormente fueron secadas bajo condiciones de laboratorio (dentro de una estufa Lindberg Blue $\mathrm{M}{ }^{\circledR}$ a $40^{\circ} \mathrm{C}$ por 48 horas). Posteriormente las ramas y hojas de las diferentes especies de plantas se pulverizaron en un molino tipo Willey con criba de $2 \mathrm{~mm}$, en el Laboratorio de Ecología Animal del Centro de Investigaciones Biológicas del Noroeste (CIBNOR) S.C., en la Paz, B.C.S., México.

La calidad nutricional de las especies forrajeras se obtuvo a partir de los análisis químicos proximales correspondientes, en el laboratorio de análisis químico proximal del CIBNOR. El contenido de cenizas (aporte de minerales, los cuáles son indispensables en los rumiantes para el funcionamiento de todos los procesos bioquímicos y para la microflora ruminal, Ramírez-Lozano, 2004), se determinó mediante incineración a $500^{\circ} \mathrm{C}$ (AOAC, 1990), la proteína cruda (PC) se obtuvo utilizando un destilador Kjeldahl $(\mathrm{N} \times$ 6,25) (AOAC 1990). La evaluación de la fibra detergente ácida (FDA) y fibra detergente neutra (FDN) que son utilizadas para la predicción de la calidad, digestibilidad y valor energético del forraje (Van Soest et al., 1991), se realizó a partir de un procedimiento no secuencial, con un analizador de fibras ANKOM ${ }^{\circledR}$ (Goering \& Van Soest, 1970; Van Soest et al., 1991). El extracto etéreo (EE) que determina el aporte de aceites y grasas esenciales, se obtuvo mediante el método de Soxhlet (AOAC, 1990), el cual consiste en la extracción de esos elementos con éter etílico a través del extractor SOXHLET ${ }^{\circledR}$. Para conocer el porcentaje de Digestibilidad in vitro de la Materia Seca (DIVMS) se utilizó la fórmula propuesta por Caddel \& Allen (2000) y Schroeder (2012), a partir de la ecuación: \% DIVMS = 88.9 - $(0.779 * \%$ FDA $)$.

Análisis de datos. Comparamos los valores de cada uno de los nutrientes entre años, estaciones, especies consumidas y formas de vida, debido a que los datos no siguieron una distribución normal, se utilizó la prueba de Kruskal-Wallis $(\mathrm{H})$. Datos con un valor de probabilidad asociado $\leq 0.05$ fueron considerados significativos.

El análisis de selección de especies forrajeras, se determinó a partir de dos indicadores:

1. Índice de Ivlev (ISI) (Strauss 1979), que relaciona de manera sencilla las proporciones usadas de cada especie vegetal y su disponibilidad en el hábitat: $I S I=\frac{\left(r_{i}-n_{i}\right)}{r_{i}+n_{i}}$, donde $r_{i}$ es el porcentaje de la especie $i$ en la dieta y $n_{i}$ es el porcentaje de la especie $i$ en la vegetación disponible. De acuerdo con el criterio de Stuth (1991) las especies seleccionadas son las que presentan valores mayores a 0.35.

2. A partir de una prueba de bondad de ajuste de $\chi^{2}$, en el que se evaluaron diferencias significativas entre la utilización esperada de cada uno de los tipos de vegetación (basada en los porcentajes de disponibilidad) y las observaciones de uso (porcentaje de consumo), se examinó la hipótesis de selección de uso al azar. Cuando la prueba de $\chi^{2}$ muestra diferencias entre el uso esperado y el observado, se calculan los intervalos de confianza al 95\% Bonferroni (Marcum \& Loftgarden, 1980; Aho \& Bowyer, 2015). Dichos intervalos, se usaron con la finalidad de determinar qué especies vegetales están siendo seleccionadas, es decir, cuando el uso observado está por fuera del intervalo calculado para el valor observado (Marcum \& Lofstgarden, 1980; Byers et al., 1984; Aho \& Bowyer, 2015).

Utilizamos el análisis de componentes principales (ACP) con los datos de los años y estaciones para examinar las relaciones entre las variables químicas de los forrajes consumidos y las cuatro etapas fisiológicas de los animales (a las que se les asignó un número), para evaluar su potencial como variables explicativas de las especies mayormente seleccionadas e importantes por sus características nutrimentales. 
Se incluyeron cinco variables químicas de los forrajes (los porcentajes se transformaron a coseno al cuadrado), además de las especies encontradas en el índice de Ivlev en cada estación de los dos años. Los análisis estadísticos se realizaron mediante el programa XLSTAT-ecology versión 17.1. Microsoft ${ }^{\circledR}$ Excel® 2016.

\section{RESULTADOS}

Los resultados del análisis químico proximal de las especies consumidas por los borregos se presentan en el Anexo 1. De manera general, se observó que los valores nutrimentales de mayor porcentaje como las cenizas (minerales), la proteína cruda y el extracto etéreo (aceites esenciales), se encontraron principalmente en los árboles, seguidos de los arbustos y las herbáceas.

Con excepción de la FDN ( $\mathrm{H}=22.038)$, no se encontraron diferencias significativas de los nutrientes entre años $(H=0.312)$ ni entre estaciones $(H=0.487)$. Respecto a los valores de los nutrientes por formas de vida en cada estación, se encontraron diferencias significativas en la FDN de las arbustivas de primavera $(\mathrm{H}=0.022)$ y verano $(\mathrm{H}=0.033)$, y las herbáceas de invierno $(\mathrm{H}=0.042)$, así como para los componentes de ceniza (0.011) de los arbustos en invierno.

Con respecto a la fluctuación de nutrientes por especie, el mayor valor de DIVMS se presentó en Viscainoa geniculata (71.9\%) durante primavera, y en Opuntia cholla (71.7\%) en el invierno. Los porcentajes de ceniza más altos durante la primavera correspondieron a Buddleja corrugata (11.7 \%), y a Lysiloma candida $(9.1 \%)$ en el verano. Respecto a la PC, la mayor concentración se encontró en primavera en L. candida l (22.3 \%), y en Fouquieria diguetii en el invierno (16.4\%). La FDA fue más alta durante la primavera en Condalia globosa (56.9\%) y en Krameria parvifolia l (50.2\%) en el otoño. En cuanto a los porcentajes de la FDN, los valores mayores correspondieron a dos especies de pastos de primavera e invierno; Aristida adscensionis (80.2\%) y Bouteloua aristidoides (78.4\%). Por último, el EE fue más alto en primavera en Bursera epinnata (5.3\%) y en invierno en Croton caboencis (5.7\%).

De acuerdo al índice de Ivlev, los borregos seleccionaron cinco especies arbustivas en 2010, Bourreria sonorae, Caesalpinea placida, Ditaxis lanceolata, K. parvifolia y Larrea tridentata, y una en 2011, Melochia tomentosa. Solo dos árboles, B. epinnata en 2010 y Olneya tesota en 2011, estuvieron presentes en las diferentes estaciones de los dos años de estudio, con valores mayores a 0.35.

La prueba Bonferroni mostró que los borregos seleccionaron diez especies forrajeras (cinco por año): ocho arbustivas, un árbol y una herbácea (Cuadro 1). En 2010, la selección fue más evidente durante otoño e invierno, mientras que en 2011 se observó durante las cuatro estaciones. Por su parte, con los dos índices se establecieron tres especies por año (Bourreria sonorae, Caesalpinea placida y Melochia tomentosa en 2010; B. epinnata, Caesalpinea placida y Larrea tridentata en 2011) como las de mayor selección durante las diferentes estaciones. C. placida, fue la única especie que se seleccionó en los dos años, particularmente durante otoño e invierno.

En la gráfica del ACP (Fig. 3) tras rotación Varimax, es posible visualizar la asociación de las diferentes especies forrajeras preferidas en cada estación y año, con los nutrientes de mayor importancia durante las diferentes etapas fisiológicas de los borregos. Los dos primeros componentes acumularon el $72.45 \%$ de la variación de los datos. Se observa que las cenizas y PC, se asocian con tres especies de forrajes: la arbustiva C . placida en primavera, otoño e invierno de 2010, y los árboles B. epinnata y $O$. tesota en el invierno de 2011. El EE se asoció con B. epinnata y B. odorata en otoño de 2010, mientras que B. epinnata, O. tesota y la arbustiva $M$. tomentosa, en primavera e invierno del 2011. Otro grupo particular asociado con la FDN, lo conformaron dos especies arbustivas leñosas: $B$. sonorae durante primavera, verano y otoño de 2010 y L. tridentata solo en el invierno de 2011. 
En la misma figura, respecto a la asociación de especies y sus nutrientes con los diferentes eventos fisiológicos de los borregos, se aprecia que durante las etapas uno (gestación), tres y cuatro (primera y segunda etapas de nacimientos y lactancia) se asociaron al consumo de especies con valores medios de PC y valores altos de cenizas, EE y FDA, mientras que solo durante la segunda etapa de gestación (etapa dos), el consumo de forrajes se asoció con la FDN.

Cuadro 1. Intervalos de confianza de Bonferroni, que expresan el nivel de selección de las especies forrajeras por parte de los borregos y el contenido nutricional en las diferentes estaciones de los dos años. UE = Uso esperado, UO = uso observado, LI = límite inferior, LS = límite superior.

\begin{tabular}{|c|c|c|c|c|c|c|c|c|c|c|c|c|}
\hline \multirow[b]{2}{*}{ Año 2010} & \multirow[b]{2}{*}{ UE } & \multirow[b]{2}{*}{ UO } & \multirow[b]{2}{*}{ LI } & \multirow[b]{2}{*}{ LS } & \multicolumn{6}{|c|}{$\%$} & \multirow{2}{*}{$\begin{array}{l}\text { Forma de } \\
\text { vida }\end{array}$} & \multirow[t]{2}{*}{ Familia } \\
\hline & & & & & Ceniza & $\mathrm{PC}$ & FDA & FDN & $\mathrm{EE}$ & DIVMS & & \\
\hline \multicolumn{13}{|l|}{ Bourreria sonorae } \\
\hline Otoño & 0.142 & 0.582 & -1.010 & 0.131 & 3.0 & 5.7 & 40.0 & 53.2 & 1.7 & 57.8 & $\mathrm{Ab}$ & Boraginaceae \\
\hline \multicolumn{13}{|c|}{$\begin{array}{l}\chi^{2}=18.30 \text { g.l. }=3 \mathrm{p}<0.05 \\
\text { Caesalpinia placida }\end{array}$} \\
\hline Otoño & 0.374 & 0.586 & -0.596 & 0.171 & 6.9 & 9.7 & 45.5 & 44.9 & 2.6 & 53.5 & $\mathrm{Ab}$ & Leguminosae \\
\hline Invierno & 0.215 & 0.278 & -0.395 & 0.269 & 7.0 & 10.1 & 47.4 & 45.0 & 2.8 & 52.0 & & \\
\hline \multicolumn{13}{|c|}{$\chi^{2}=12.97$ g.l. $=3 p<0.05$} \\
\hline Invierno & 0.203 & 0.370 & -0.618 & 0.283 & 5.8 & 8.2 & 40.7 & 48.2 & 4.2 & 57.2 & $\mathrm{H}$ & Malvaceae \\
\hline \multicolumn{13}{|c|}{$\chi^{2}=9.82$ g.l. $=3 \mathrm{p}<0.05$} \\
\hline \multicolumn{13}{|c|}{ Melochia tomentosa } \\
\hline Otoño & 0.098 & 0.448 & -0.720 & 0.021 & 6.4 & 9.8 & 32.3 & 45.0 & 1.8 & 63.8 & $\mathrm{Ab}$ & Sterculiaceae \\
\hline \multicolumn{13}{|c|}{$\begin{array}{l}\chi^{2}=7.91 \text { g.l. }=3 \mathrm{p}<0.05 \\
\text { Ruellia californica }\end{array}$} \\
\hline Invierno & 0.271 & 0.419 & -0.461 & 0.165 & 10.3 & 16.1 & 28.4 & 44.6 & 1.4 & 66.8 & $\mathrm{Ab}$ & Acanthaceae \\
\hline \multicolumn{13}{|c|}{$\chi^{2}=15.38$ g.l. $=3 p<0.05$} \\
\hline \multicolumn{13}{|l|}{ Año 2011} \\
\hline \multicolumn{13}{|l|}{ Bursera epinnata } \\
\hline Primavera & 0.067 & 0.322 & -0.610 & 0.099 & 7.7 & 11.3 & 40.0 & 46.7 & 5.3 & 57.7 & $\mathrm{Ab}$ & Burseraceae \\
\hline Invierno & 0.117 & 0.282 & -0.558 & 0.228 & 6.8 & 10.9 & 41.2 & 47.3 & 4.3 & 56.8 & & \\
\hline \multicolumn{13}{|c|}{$\begin{array}{l}\chi^{2}=15.35 \text { g. } 1 .=3 \mathrm{p}<0.05 \\
\text { Caesalpinia placida }\end{array}$} \\
\hline Otoño & 0.212 & 0.629 & -0.648 & 0.190 & 5.5 & 9.0 & 43.7 & 56.4 & 1.2 & 54.9 & $\mathrm{Ab}$ & Leguminosae \\
\hline \multicolumn{13}{|c|}{$\chi^{2}=56.21$ g. $1 .=3 \mathrm{p}<0.05$} \\
\hline Primavera & 0.251 & 0.48 & -0.567 & 0.10 & 6.8 & 16.4 & 28.7 & 40.9 & 2.5 & 66.6 & $\mathrm{Ab}$ & Fouquieriaceae \\
\hline \multicolumn{13}{|c|}{$\begin{array}{l}\chi^{2}=4.95 \text { g. } 1 .=3 \mathrm{p}<0.05 \\
\text { Jatropha cuneata }\end{array}$} \\
\hline Verano & 0.262 & 0.416 & -0.460 & 0.150 & 6.8 & 7.8 & 40.8 & 48.3 & 3.1 & 57.1 & $\mathrm{Ab}$ & Euphorbiaceae \\
\hline \multicolumn{13}{|c|}{$\begin{array}{l}\chi^{2}=20.95 \text { g. } 1 .=3 \mathrm{p}<0.05 \\
\text { Larrea tridentata }\end{array}$} \\
\hline Primavera & 0.101 & 0.482 & -0.68 & -0.080 & 5.7 & 7.6 & 41.7 & 52.7 & 1.3 & 56.5 & $\mathrm{Ab}$ & Zygophyllaceae \\
\hline Invierno & 0.134 & 0.343 & -0.517 & 0.100 & 4.3 & 6.3 & 39.3 & 55.2 & 1.2 & 58.3 & & \\
\hline$\chi^{2}=17.63$ g.l. $=3$ & & & & & & & & & & & & \\
\hline
\end{tabular}

\section{DISCUSIÓN}

Bajo el contexto de la selección de los alimentos, las especies animales en general, han evolucionado en relación a diferentes presiones ecológicas, como la abundancia, distribución y calidad de 
su alimento y los depredadores. Por lo que dichas presiones selectivas, son el resultado de la adaptación, donde solo persisten aquellos individuos que se han alimentado de manera óptima, es decir, consumen aquellos alimentos de los que se obtiene el máximo beneficio con el mínimo costo (Hawkes \& O’Connel, 1992).

Mc Arthur y Pianka (1966), detallan que un animal incorpora alimentos en su dieta a partir de su rendimiento energético. Así que dicha incorporación, indica que a medida que disminuyen los recursos de más alto contenido nutricional, se incorporan en la dieta aquellos de menor valor nutrimental o rendimiento. Es decir, los animales pueden escoger los alimentos más beneficiosos entre los accesibles, ser más selectivos cuando hay abundancia e ignorar aquellos de poco beneficio, no importa qué tan comunes sean.

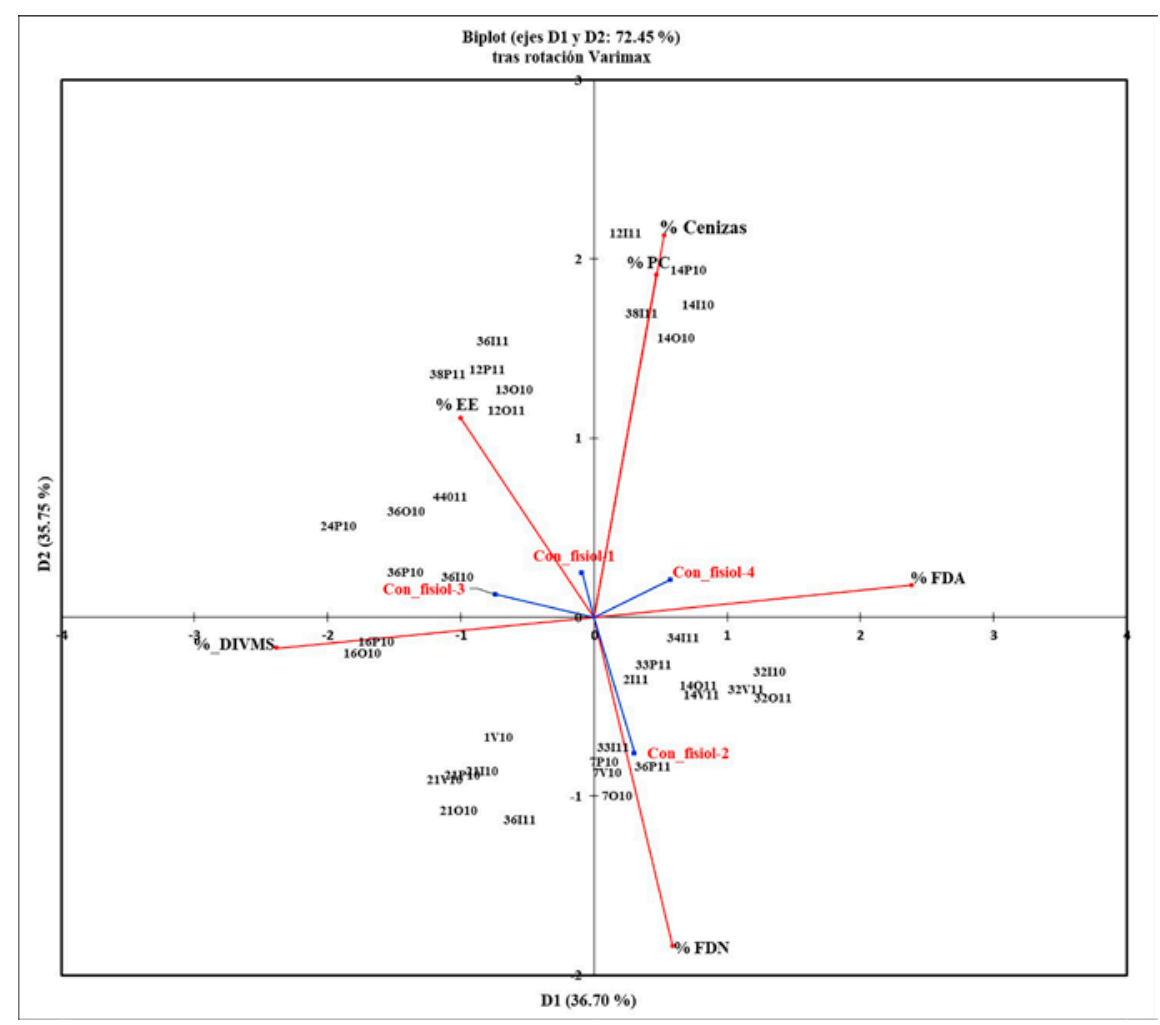

Figura 3. Análisis de Componentes Principales tras rotación Varimax, que presenta la agrupación de las especies seleccionadas (Índice de Ivlev) con sus respectivos valores nutrimentales. Los valores de números y letras corresponden a lo siguiente: la primera letra es la especie de planta, la segunda letra es para la estación, ya sea primavera, verano, etc. y los números corresponden al año de estudio, 2010 o 2011.

Por otro lado, Plata et al. (2009) explican que la selección de los alimentos está dada por la palatabilidad, la cual es definida como la característica de un alimento que estimula una respuesta selectiva de un animal y es un factor determinante en el consumo de las especies vegetales, lo cual tiene implicaciones importantes en la elección de alimentos. Esta palatabilidad de las plantas depende de los cambios estacionales que provocan que varíen en su abundancia, estado de crecimiento y características nutrimentales. 
Una de las posibles razones de la selección de la dieta del borrego cimarrón, es que éste ha evolucionado para aprovechar al máximo los nutrientes presentes en el forraje que consume, para que su tasa reproductiva sea mayor y de esta manera aumenten sus poblaciones (Pelletier \& Festa-Bianchet, 2004).

Los nutrientes aportados por las especies que consumen los borregos, como la proteína cruda, los minerales y el extracto etéreo, no presentaron cambios significativos en los dos años y estaciones muestreadas, con excepción de los porcentajes de FDN. El aumento en los valores de FDN, puede obedecer a la elevada temperatura ambiental y a la falta de lluvia, factores que deshidratan y deterioran la vegetación, sobre todo a las especies de pastos, volviéndolas más fibrosas y difíciles de digerir (Wilmshurst et al., 1995).

En este estudio, Aristida adscensionis y Bouteloua aristidoides se consideran poco nutritivas, ya que presentaron un contenido de FDN de 61.3 \% en 2010 y de $79.6 \%$ en 2011 que de acuerdo con Hoffman et al. (2007), valores altos de FDN de 55 a $75 \%$ se consideran de baja digestibilidad. Esto concuerda con Ramírez-Lozano et al. (2008) quienes mencionan que en las zonas áridas del norte de México, los pastos tienen alto contenido de elementos estructurales (celulosa y hemicelulosa) de baja digestibilidad, lo cual los limita en cuanto a su valor nutrimental, y por lo tanto, son menos consumidos por los rumiantes silvestres y domésticos.

Niwinska (2012) señala que el sistema digestivo de los rumiantes presenta adaptaciones funcionales y anatómicas que les ha permitido obtener energía disponible a partir de material fibroso de algunas plantas. Particularmente en el caso del borrego cimarrón, Miller \& Gaud (1989) explican que tanto los grandes molares (responsables de romper el alimento en pequeñas partículas), como el gran volumen de su retículo-rumen (que permiten un paso lento del alimento), son consideradas adaptaciones para el consumo de especies vegetales con alto contenido de celulosa de difícil digestión.

Krausman et al. (1989) añaden que la composición de la microflora ruminal de los borregos permite que se incremente el tiempo de fermentación del forraje en el rumen, mejorando así la digestibilidad de alimentos de baja calidad. Otra explicación al consumo de fibras es la de Van Soest et al. (1992), quienes señalan que los rumiantes consumen alimentos con suficiente cantidad de fibra debido a que ésta estimula la rumia y con ello la secreción de cantidades abundantes de saliva, permitiendo regular la acidez del rumen y los procesos de fermentación, lo cual en forma general mantiene en buen estado la salud del rumen de estos animales. Por otro lado, Weiss (1993) especifica que forrajes con niveles menores a 30\% de FDN, pueden causar problemas metabólicos en los rumiantes.

Goodson et al. (1991) explican que la fenología de las plantas se ve reflejada en la selección de las especies que utilizan los borregos, y en el caso de este estudio, las especies arbustivas fueron las de mayor consumo debido a que presentaron follaje verde la mayor parte del año, beneficiadas por la humedad proveniente del Golfo de California (León de la Luz et al., 1996). Esto puede corresponder con la dominancia de estas especies en la zona de estudio.

El predominio de especies arbustivas en la dieta de los borregos, también puede obedecer a que en la mayor parte del año, éstas tuvieron porcentajes aceptables de proteína cruda, con un promedio de 8.5\% durante los dos años del estudio. Mazaika et al. (1992) y Memmott et al. (2011) explican que los arbustos de zonas áridas destinan reservas de nutrientes durante su crecimiento para la formación de nuevos tejidos y ramas tiernas, por tal razón, contienen mayor cantidad de proteína cruda, a diferencia de algunas herbáceas y gramíneas. Así mismo, los arbustos mantuvieron cantidades significativas de proteína cruda y otros nutrientes durante todo el año. 
Una posible causa por la que los arbustos mantienen niveles importantes de proteína cruda a lo largo del año, independientemente de la precipitación, podría ser la presencia de fósforo en el área de estudio, debido a que esta zona es rica en fosforita (roca sedimentaria con alta concentración de minerales fosfatados). Esto puede estar relacionado con lo reportado por Hoffman et al. (2007), quienes encontraron que particularmente en las formas arbustivas, niveles altos de proteína están asociados con altos niveles de fósforo. Marschner (1993) explica que el fósforo favorece una serie de funciones metabólicas y es un nutriente esencial para el crecimiento y el desarrollo de las plantas.

Mediante los dos indicadores de preferencia utilizados, se encontró que Caesalpinia placida fue la especie principalmente seleccionada durante otoño e invierno de los dos años. Esta especie presentó niveles medios de nutrientes, con porcentajes de ceniza (6.9 y 7.9\%), PC (9.7 y 10), y EE (2.6 y 2.8\%), así como niveles bajos de las fibras FDA (44.9\%) y FDN (45.0\%) constantes durante el año. La alta selección de este arbusto, puede estar relacionada con su constante presencia en el hábitat, lo cual puede obedecer a que su floración se presenta a lo largo de todo el año (León de la Luz et al., 1996).

Foroughbakhch et al. (2009) refieren que los árboles contienen altos contenidos de compuestos fenólicos, lo cual afecta al rumen y por lo tanto, no son digeribles. Lo anterior podría explicar por qué los árboles fueron escasamente utilizados, según el Índice de Ivlev. No obstante, los borregos consumieron cuatro especies: Lysiloma candida, Olneya tesota, Prosopis glandulosa y Bursera epinnata; las tres primeras contienen una gran cantidad de proteína cruda (hasta $22.3 \%$ ), la cual se mantiene a lo largo del año. Esto puede deberse a que cuando el forraje escasea y es pobre en nutrientes, los ungulados silvestres se ven forzados a cambiar la composición de su dieta (Hoffman, 1989; Owen-Smith, 1988) para satisfacer los requerimientos de algunos minerales esenciales, consumiendo especies ricas en nutrientes, incluso algunas que normalmente son evitadas debido a la presencia de compuestos secundarios (Robbins et al., 1987; Holt et al., 1992; Wagner \& Peek, 2006).

Con el ACP se encontró una agrupación de especies seleccionadas: a) C. placida, B. epinnata - O. tesota y b) B. epinnata - B. odorata - O. tesota - M. tomentosa c) Bourreria sonorae - Larrea tridentata que presentan altos porcentajes de minerales (ceniza), proteína cruda, extracto etéreo y FDN. Estas especies se encontraron asociadas con tres etapas de gran importancia para los borregos: gestación (finales de verano y todo el otoño) y la primera y segunda etapa de nacimientos y lactancia (que ocurren durante todo el invierno y primavera). Estos resultados coinciden con los de Krausman et al. (1989), quienes encontraron que la mayor parte de las especies consumidas por los borregos de Arizona, tuvieron altos porcentajes de proteína cruda, altamente relacionada con las épocas de nacimientos y lactancia.

El borrego cimarrón, particularmente las hembras durante la gestación y la lactancia, buscan especies con altos contenidos de proteína cruda debido a que necesitan producir leche de buena calidad para la sobrevivencia de los corderos (Goodson et al., 1991; Holt et al., 1992); esto concuerda con Martínez (2010), quien menciona que el consumo de ciertos tipos de alimento por ungulados silvestres, está relacionado con las necesidades energéticas y nutrimentales, de acuerdo a su estado fisiológico. Hawkes \& Connel (1992) señalan que cuando el alimento es abundante, los borregos consumen preferentemente especies con altos contenidos de nutrientes. Por su parte, si las condiciones del hábitat son desfavorables y con poca disponibilidad de alimento, éstos suelen incorporar a su dieta, incluso a especies de menor calidad nutricional. Guerrero-Cárdenas et al. (2016) encontraron que el borrego cimarrón seleccionó particularmente las especies arbustivas B. sonorae, C. placida, M. tomentosa y $L$. tridentata, las cuales junto con el árbol B. epinnata, presentaron porcentajes medios de proteína cruda de $10.5 \%$, extracto etéreo de $2.3 \%$ y minerales de $5.5 \%$, siendo estos nutrientes esenciales durante sus diferentes etapas fisiológicas.

En zonas desérticas donde las condiciones climáticas son frecuentemente adversas durante gran parte del año (pocos días de lluvia, calor excesivo y recurrentes periodos de sequía), la estrategia 
alimentaria de los borregos consiste en consumir los forrajes palatables que les permitan sobrevivir y tener crías (Monson \& Sumner, 1980). En este estudio, se observó un comportamiento especialista por parte de los borregos, los cuales a pesar de la poca disponibilidad de alimento y la escasez de lluvias, pudieron desplazarse hacia otros sitios en busca de alimento de buena calidad. Schroeder et al. (2010) mencionan que los borregos, sobre todo las hembras, se alimentan cerca de acantilados y zonas rocosas con menor abundancia de forraje, pero de mayor calidad, ya que éstas coinciden con la selección del terreno de escape y menor riesgo de depredación (Monson \& Sumner, 1980; Tarango \& Krausman, 1997; Sandoval et al., 2014).

Los borregos de la Sierra El Mechudo consumieron especies vegetales de buena y mediana calidad, debido a que fueron más palatables. Por otro lado, el equilibrio entre aportes medios de proteína y altos niveles de fibra, proporciona, particularmente durante algunas etapas fisiológicas, una buena salud de la microflora ruminal, permitiendo que los animales se nutran óptimamente, es decir, que obtengan el mejor rendimiento del alimento seleccionado con el menor costo energético (Poppi \& McLennan, 1995; Niwinska, 2012).

\section{CONCLUSIONES}

Los resultados presentados en este estudio, son los primeros que exponen los valores nutrimentales de las especies vegetales que consume el borrego cimarrón en la Península de Baja California a lo largo del año, ya que anteriormente solo se habían enlistado las especies de la dieta.

Aunque los árboles cuentan con altos porcentajes de nutrientes, sobre todo de proteína cruda, éstos no son seleccionados debido probablemente a las altas concentraciones de compuestos fenólicos que dañan el rumen de los borregos y son menos digeribles.

Las especies arbustivas presentaron cantidades medias y altas de nutrientes (sobre todo de proteína), los cuales se mantienen constantes durante todas las estaciones, y son esenciales durante las etapas de mayor demanda de energía, principalmente en las épocas de nacimiento y lactancia. La selectividad de los borregos por especies arbustivas, se debe principalmente a la calidad nutricional de éstas, siendo importante también, la palatabilidad y su disponibilidad a lo largo del año, principalmente en épocas críticas. Particularmente, el arbusto Caesalpinia placida fue la especie principalmente seleccionada durante los dos años de estudio, presentando una buena calidad nutricional.

Las especies vegetales que se analizaron, pueden ser utilizadas como indicadoras de la calidad del hábitat, debido a que son especies perennes que siempre están disponibles, presentando buena calidad nutrimental; además de ser utilizadas por otras especies de fauna silvestre y doméstica que se distribuyen en la Sierra El Mechudo.

La evaluación de elementos bióticos del hábitat, como son las especies vegetales que constituyen la dieta del borrego, con sus valores nutrimentales y disponibilidad, son esenciales para obtener las bases de un programa de manejo que promueva la conservación del hábitat y el uso sustentable de la población del borrego cimarrón en la Sierra El Mechudo.

Agradecimientos. Agradecemos el apoyo económico de los proyectos CONABIO (2009-GN024) y CIBNOR PC0.24. Al comisariado del ejido Ley Federal de Aguas No. 2 y a la empresa Roca Fosfórica Mexicana, por brindarnos la facilidad de acceder a la zona del Junco. Agradecemos el apoyo de los técnicos de campo y laboratorio: Abelino Cota, Marcos Acevedo, Agustín Argueta y Joaquín Rivera. Un particular agradecimiento a los revisores anónimos, por sus acertadas observaciones al manuscrito. 


\section{LITERATURA CITADA}

Álvarez-Cárdenas, S., Galina-Tessaro, P., Díaz-Castro, S., Guerrero-Cárdenas, I., CastellanosVera, A., Mesa-Zavala, E. (2009) Evaluación de elementos estructurales del hábitat del borrego cimarrón en la Sierra del Mechudo, Baja California Sur, México. Tropical Conservation Science, 2, 189-203.

Aho, K., Bowyer, R. T. (2015) Confidence intervals for ratios of proportions: implications for selection ratios. Methods in Ecology and Evolution, 6, 121-132.

AOAC. (1990) Official methods of analysis. Edition: 15th. Association of Official Analytical Chemist. Arlington, Virginia, EEUU. 1213 pp.

Arriaga, L., Espinoza, J. M., Aguilar, C., Martínez, E., Gómez, L., Loa, E. (Coords.) (2000) Regiones terrestres prioritarias de México. Comisión Nacional para el Conocimiento y Uso de la Biodiversidad. México.

Bolen, E.G., Robinson, W.L. (2002) Wildlife Ecology and Management. Edition: 5th. Prentice Hall Inc. Englewood Cliffs, New Jersey, EEUU. 350 pp.

Byers, C. R., Steinhorst, R. K., Krausman, P. R. (1984) Clarification of a technique for analysis of utilization-availability data. Journal of Wildlife Management, 48, 1050-1053.

Caddel, J., Allen, E. (2000) Forage Quality Interpretations. Division of Agriculture Sciences and Natural Resources. Oklahoma University. EEUU. 10 pp.

Corriale, M. J., Arias, S. M., Quintana, R. D. (2011) Forage quality of plant species consumed by capibaras (Hydrochoerus hydrochaeris) in the Paraná River Delta, Argentina. Rangeland Ecology \& Management, 64, 257-263.

Cunningham, S. C. (1989) Evaluation of bighorn sheep habitat. Pp. 135-160. In: R. M. Lee (Editor). The desert bighorn sheep in Arizona. Arizona Game and Fish Department. Phoenix. EEUU.

DeYoung, R. W., Hellgren, E. C., Fulbrigth, T. E., Robbins, W. F., Humphreys, I. D. (2000) Modeling nutritional carrying of translocated desert bighorn sheep in western Texas. Restoration Ecology, 8, 57-65.

SEMARNAT. (2010) Norma Oficial Mexicana NOM-059-SEMARNAT-2010, Protección ambiental Especies nativas de México de flora y fauna silvestres -Categorías de riesgo y especificaciones para su inclusión, exclusión o cambio -Lista de especies en riesgo. Diario Oficial de la Federación: 30/12/2010.

Escobar-Flores, J. G., Álvarez-Cárdenas, S., Valdez, R., Torres Rodríguez, J., Díaz-Castro, S., Castellanos-Vera, A., Martínez-Gallardo, R. (2015) Detección de las preferencias de hábitat del borrego cimarrón (Ovis canadensis cremnobates) en Baja California, mediante técnicas de teledetección satelital. Therya, 6, 519-534.

Festa-Bianchet, M. (1988) Seasonal range selection in bighorn sheep: conflicts between forage quality, forage quantity, and predator avoidance. Oecologica, 75, 580-586.

Foroughbakhch, R., Hernández, P. J., Alvarado, V. M., Céspedes, C. E., Rocha, E. A., Cárdenas, A. M. (2009) Leaf biomass determination on woody shrub species in semiarid zones. Agroforestry Systems, 77, 181-192.

García, E. (1988) Modificaciones al sistema de clasificación climática de Köeppen. Instituto de Geología, UNAM, México, D.F.

Geist, V. (1971) Mountain Sheep: A Study in Behavior and Evolution. The University of Chicago Press. Chicago. 383 pp.

Goering, H. K., Van Soest, P. J. (1970) Forage fiber analyses (apparatus, reagents, procedures and some applications). Agriculture Handbook. No. 379. ARS, USDA, Washington, D.C. 20 pp.

Goodson, N. L., Stevens, D. R., Bailey, J. A. (1991) Winter-spring foraging ecology and nutrition of bighorn sheep on montane range. Journal of Wildlife Management, 3, 422-433. 
Guerrero-Cárdenas, I., Gallina, S., Corcuera, P., Álvarez-Cárdenas S., Ramírez-Orduña R. (2016) Diet composition and selection of the bighorn sheep (Ovis canadensis) in Sierra El Mechudo, Baja California Sur, Mexico. Therya, 7, 423-438.

Hawkes, K., O’Connell, J. F. (1992) On optimal foraging models and subsistence transitions. Current Anthropology, 33, 63-65.

Hanley, T. A. (1997) A nutritional view of understanding and complexity in the problem of diet selection by deer (Cervidae). Oikos, 79, 209-218.

Hobbs, N. T., Baker, D. L., Bear, G. D., Bowden, D. C. (1996) Ungulate grazing in sagebrush grassland: Mechanisms of resource competition. Ecological Applications, 61, 200-217.

Hofmann, R. R. (1989) Evolutionary steps of ecophysiological adaptation and diversification of ruminants: a comparative view of their digestive system. Oecologia, 78, 443-457.

Hoffman, P. C., Lundberg, K. M., Shaver, R. D., Contreras-Goeva, F. E. (2007) Use of digestibility of NDF in the formulation of rations. Focus on Forage, 63, 1-5.

Holt, B., Miller, W. H., Wakelling, B. F. (1992) Composition and quality of mountain sheep diets in the Superstition Mountains, Arizona. Desert Bighorn Council Transactions, 36, 36-40.

Jiménez, S. D., Hernández, M. C. (2010) Programa de conservación del borrego cimarrón (Ovis canadensis weemsi) en Baja California Sur, México. Galemys, 22 (nº especial), 447-468.

León de la Luz, J. L., Coria, B. R., Cruz, E. M. (1996) Fenología floral de una comunidad áridotropical de Baja California Sur, México. Acta Botánica Mexicana, 35, 45-64.

Krausman, P. R., Leopold, B. D., Seegmiller, R. F., Steven, G. T. (1989) Relationships between desert bighorn sheep and habitat in western Arizona. Wildlife Monographs, 102, 3-66.

Martínez, T. (2010) Selección y estrategia alimentaria de los machos, hembras y jóvenes de cabra montés (Capra pirenaica Schinz, 1838) en el suroeste de España. Galemys, 22, 483-515

Marcum, C. L., Loftsgaarden, D. (1980) A nonmapping technique for studying habitat preferences. Journal of Wildlife Management, 44, 963-968.

Marschner, H. (1993) Mineral nutrition of higher plants. Academic Press Ltd., Harcourt Brace \& Co. Publishers, London. 889 pp.

Mazaika, R., Krausman, P. R., Etchberger, R. C. (1992) Nutritional carrying capacity of desert bighorn sheep habitat in the Pusch Ridge Wilderness, Arizona. Southwestern Naturalist, 37, 372-378.

McArthur, R. H., Pianka, E. R. (1966) On the optimal use of a patchy environment. American Naturalist, 100, 603-609.

Memmott, K. L., Anderson, V. J., Fugal, R. (2011) Seasonal dynamics of forage shrub nutrients. Rangelands, 6, 12-16.

Mertens, D. R. (1987) Predicting intake and digestibility using mathematical models of ruminal function. Journal of Animal Science, 64, 1548-1558.

Miller, G. D., Gaud, W. S. (1989) Composition and variability of desert bighorn sheep diets. Journal of Wildlife Management, 53, 597-606.

Monson, G., Sumner, L. (1980) The desert bighorn; its life history, ecology and management. The University of Arizona Press, Tucson. 365 pp.

Niwińska, B. (2012) Digestion in Ruminants. Pp. 245-258. In: Chuan-Fa Chang (Eds). CarbohydratesComprehensive Studies on Glycobiology and Glycotechnology. InTech, DOI: 10.5772/51574. Available from: https://www.intechopen.com/books/carbohydrates-comprehensive-studies-onglycobiology-and-glycotechnology/digestion-in-ruminants

Owen-Smith, R. N. (1988) Megaherbivores. The influence of very large body size on Ecology. Cambridge Studies in Ecology, Cambridge University Press. 369 pp.

Pelletier, F., Festa-Bianchet, M. (2004) Effects of body mass, age, dominance and parasites load on foraging time of bighorn ram Ovis canadensis. Behavior Ecology and Sociobiology, 6, 546-551.

Plata, F. X, Ebergeny, S., Resendiz, J. L., Villarreal, O., Bárcena, R., Viccone, J. A., Mendoza, G. D. (2009) Palatabilidad y composición química de alimentos consumidos en cautiverio por el 
venado cola blanca de Yucatán (Odocoileus virginianus yucatanensis). Archivos Médicos Veterinarios, 41, 123-129.

Poppi, D. P., Mclennan, S. R. (1995) Protein and energy utilization by ruminants at pasture. Journal of Animal Science, 73, 278-290.

Robbins, C. T., Mole, S., Hagerman, A. E., Hanley, T. A. (1987) Role of tannins in defending plants against ruminants: Reduction in dry matter digestion? Ecology, 68, 1606-1615.

Ramírez-Lozano, R. G. (2004) Nutrición del venado cola blanca. Publicaciones Universidad Autónoma de Nuevo León, San Nicolás de Los Garza, N.L., México. 66 pp.

Ramírez-Lozano, R. G., Morales-Rodríguez, R., Cerrillo-Soto, A., González-Rodríguez, H., Juárez-Reyes, A., Guerrero-Cervantes M. (2008) Forage Nutrition of Range Grasses Growing in Northeastern Mexico. Conference on International Agricultural Research for development, 1-10 pp.

SARH. (1983) Baja California Sur. Carta Sinóptica. Escala 1: 50,000. Carta No. 2. Secretaría de Agricultura y Recursos Hidráulicos.

Sandoval, A. V., Valdez, R., Espinoza, A. T. (2014) El borrego cimarrón en México. Pp. 475-501. In: R. Valdez \& J.A. Ortega (Eds.). Ecología y Manejo de Fauna Silvestre. Colegio de Postgraduados, Texcoco, Estado de México, México.

Schroeder, A., Bowyer, T. R., Bleich, V. C., Stephenson, T. R. (2010) Sexual segregation in Sierra Nevada bighorn sheep, Ovis canadensis sierrae: Ramifications for conservation. Arctic, Antarctic, and Alpine Research, 4, 476-489.

Strauss, R. E. (1979) Reliability estimates for Ivilev's index, the forage ratio, and a proposed linear index of food selection. Transactions of American Fisheries Society, 108, 344-352.

Stuth, J. W. (1991) Foraging behavior. Pp. 65-83. In: R.K. Heitschmidt \& J.W. Stuth (Eds.). Grazing management. An ecological perspective. Timber Press, Portland, Oregon, EE.UU.

Smith, N., Krausman, P. (1988) Desert bighorn sheep: a guide to select management practices. Biological Report 88 (35). U.S. Fish and Wildlife Service, United States Department of the Interior, Washington D.C. 120 pp.

Tarango, L.A., Krausman, P. R. (1997) Desert bighorn sheep in Mexico. Desert Bighorn Council Transactions 41, 17.

USFWS. (2008) Peninsular bighorn sheep. Recovery Program. California Department of Fish and Wildlife. Sacramento California, USA, 130 pp.

Valdez, R., Krausman, P. R. (1999) Description, distribution and abundance of mountain sheep in North America. The University of Arizona Press, Tucson, EE.UU. 353 pp.

Van Soests, P. J., Robertson, J. B., Lewis, B. A. (1991) Methods for dietary fiber, neutral detergent fiber, and nonstarch polysaccharides in relation to animal nutrition. Journal of Dairy Science, 74, 35-83

Wargner, G. D., Peek, J. M. (2006) Bighorn sheep diet selection and forage quality in Central Idaho. Northwestern Science, 4, 246-258.

Weiss, W. P. (1993) Predicting energy values of feeds. Journal of Dairy Science, 6, 1802-1811.

Wilmshurst, J. F., Fryxell, J. M., Hudsonb R. J. (1995) Forage quality and patch choice by wapiti (Cervus elaphus). Behavioral Ecology, 2, 209-217. 
Anexo 1. Especies vegetales consumidas por los borregos, con sus respectivos valores nutricionales, en las diferentes estaciones de los años 2010 y 2011. PC = proteína cruda, FDA = Fibra detergente ácida, FDN = Fibra detergente neutra, EE = Extracto etéreo, DIVMS = Digestibilidad in vitro de la materia seca.

\begin{tabular}{|c|c|c|c|c|c|c|c|c|c|c|c|c|c|}
\hline $\begin{array}{c}\text { Especie/forma de } \\
\text { vida }\end{array}$ & ceniza & PC & FDA & FDN & $\mathrm{EE}$ & DIVMS & $\begin{array}{c}\text { Especie/forma de } \\
\text { vida }\end{array}$ & ceniza & $\mathrm{PC}$ & FDA & FDN & $\mathrm{EE}$ & DIVMS \\
\hline \multicolumn{8}{|c|}{ Primavera 2010} & \multicolumn{6}{|c|}{ Primavera 2011} \\
\hline \multicolumn{14}{|c|}{ Herbáceas } \\
\hline Abutilon sp. & 2.3 & 6.3 & 37.5 & 49.9 & 2.0 & 59.7 & Aristida adscencionis & 4.7 & 5.4 & 39.7 & 80.2 & 1.5 & 58.0 \\
\hline Aristida adscencionis & 4.2 & 6.6 & 37.4 & 52.2 & 2.5 & 59.8 & Bouteloua aristidoides & 4.8 & 4.6 & 44.4 & 78.8 & 1.3 & 54.3 \\
\hline Bouteloua aristidoides & 4.8 & 6.2 & 47.1 & 75.9 & 1.3 & 52.2 & Bouteloua sp. & 3.5 & 5.0 & 43.7 & 79.7 & 1.3 & 54.9 \\
\hline Hibiscus denudatus & 5.7 & 9.3 & 37.5 & 49.9 & 2.1 & 59.7 & $\begin{array}{l}\text { Eucnide cordata } \\
\text { Heteropogon }\end{array}$ & 4.0 & 7.7 & 38.8 & 46.8 & 2.2 & 58.7 \\
\hline Janusia californica & 6.5 & 8.6 & 35.3 & 43.0 & 2.7 & 61.4 & contortus & 3.6 & 4.7 & 40.8 & 78.0 & 1.2 & 57.1 \\
\hline Merremia aurea & 7.5 & 9.5 & 34.2 & 43.2 & 2.4 & 62.3 & Hibiscus denudatus & 5.3 & 8.7 & 40.9 & 55.7 & 2.1 & 57.1 \\
\hline Senna confinis & 7.6 & 5.6 & 44.5 & 74.5 & 1.9 & 54.3 & & & & & & & \\
\hline \multicolumn{14}{|c|}{ Arbustos } \\
\hline Baccharis salicifolia & 4.1 & 6.4 & 38.1 & 59.7 & 4.1 & 59.2 & Aeschinomene nivea & 5.7 & 9.7 & 41.7 & 55.7 & 1.9 & 56.4 \\
\hline Bebbia juncea & 6.5 & 7.6 & 40.0 & 46.9 & 2.5 & 57.7 & Baccharis salicifolia & 3.6 & 4.8 & 41.8 & 71.3 & 2.4 & 56.3 \\
\hline Buddleja corrugata & 11.7 & 15.2 & 28.3 & 32.7 & 3.5 & 66.9 & Bebbia juncea & 5.6 & 5.7 & 48.7 & 50.8 & 2.5 & 51.0 \\
\hline Caesalpinia placida & 6.9 & 7.1 & 41.5 & 44.2 & 1.5 & 56.6 & Bourreria sonorae & 4.9 & 7.9 & 36.8 & 41.7 & 2.4 & 60.2 \\
\hline Celtis reticulata & 5.3 & 5.9 & 31.3 & 45.5 & 2.3 & 64.5 & Brickellia glabrata & 4.4 & 6.0 & 38.2 & 59.3 & 2.7 & 59.2 \\
\hline Condalia globosa & 4.4 & 10.5 & 56.9 & 35.6 & 1.0 & 44.6 & Buddleja corrugata & 6.7 & 11.7 & 37.8 & 45.7 & 3.4 & 59.5 \\
\hline Croton caboensis & 5.3 & 6.9 & 26.5 & 23.5 & 5.4 & 68.3 & Caesalpinia placida & 5.8 & 9.8 & 43.7 & 57.8 & 1.2 & 54.9 \\
\hline Ditaxis. $S p$ & 3.5 & 4.5 & 36.5 & 53.7 & 2.2 & 60.4 & Calliandra californica & 4.7 & 7.9 & 36.6 & 49.9 & 1.2 & 60.4 \\
\hline \multicolumn{14}{|l|}{ Euphorbia } \\
\hline magdalenae & 8.1 & 4.5 & 28.7 & 47.2 & 2.9 & 66.5 & Condalia globosa & 4.9 & 8.3 & 45.7 & 65.3 & 3.5 & 53.3 \\
\hline Jatropha cuneata & 7.6 & 8.1 & 37.9 & 46.5 & 3.5 & 59.4 & Croton magdalenae & 4.5 & 6.0 & 37.7 & 49.8 & 2.3 & 59.5 \\
\hline Krameria parvifolia & 5.9 & 8.8 & 40.1 & 48.6 & 1.5 & 57.7 & Fouquieria diguetii & 6.8 & 16.4 & 28.7 & 40.9 & 2.5 & 66.6 \\
\hline Melochia tomentosa & 5.5 & 8.6 & 31.8 & 45.5 & 1.3 & 64.1 & Jatropha cuneata & 6.9 & 8.0 & 40.8 & 47.7 & 3.3 & 57.1 \\
\hline \multirow[t]{6}{*}{ Ruellia californica } & 9.6 & 16.0 & 26.2 & 43.4 & 2.2 & 68.5 & Krameria parvifolia & 6.6 & 4.8 & 47.3 & 47.8 & 0.6 & 52.0 \\
\hline & & & & & & & Larrea tridentata & 5.7 & 7.6 & 41.7 & 52.7 & 1.3 & 56.5 \\
\hline & & & & & & & Melochia tomentosa & 5.4 & 9.1 & 37.7 & 67.9 & 1.3 & 59.5 \\
\hline & & & & & & & Merremia aurea & 7.0 & 9.0 & 34.2 & 47.9 & 2.4 & 62.3 \\
\hline & & & & & & & Ruellia californica & 5.6 & 12.0 & 32.3 & 54.9 & 1.8 & 63.8 \\
\hline & & & & & & & Vizcainoa geniculata & 7.0 & 10.2 & 30.7 & 40.4 & 4.6 & 65.0 \\
\hline \multicolumn{14}{|c|}{ Árboles } \\
\hline Bourreria sonorae & 3.2 & 6.4 & 39.5 & 52.2 & 1.4 & 58.1 & Bursera epinnata & 7.7 & 11.3 & 40.0 & 46.7 & 5.3 & 57.7 \\
\hline Bursera epinnata & 9.0 & 12.1 & 40.6 & 46.0 & 5.1 & 57.3 & Bursera odorata & 7.5 & 12.2 & 43.4 & 51.5 & 3.8 & 55.1 \\
\hline Bursera odorata & 8.7 & 10.1 & 40.6 & 46.5 & 4.7 & 57.3 & Colubrina glabra & 4.2 & 6.6 & 39.5 & 46.0 & 2.4 & 58.1 \\
\hline \multirow[t]{3}{*}{ Lysiloma candida } & 9.0 & 22.3 & 24.5 & 42.6 & 2.1 & 69.8 & Olneya tesota & 7.9 & 15.8 & 37.4 & 45.6 & 1.6 & 59.7 \\
\hline & & & & & & & Prosopis glandulosa & 7.0 & 10.7 & 36.9 & 56.5 & 2.3 & 60.2 \\
\hline & \multicolumn{7}{|c|}{ Verano 2010} & \multicolumn{6}{|c|}{ Verano 2011} \\
\hline \multicolumn{14}{|c|}{ Herbáceas } \\
\hline Abutilon sp. & 2.5 & 5.9 & 38.9 & 45.3 & 1.8 & 58.6 & Aristida adscencionis & 4.7 & 5.8 & 39.8 & 80.0 & 1.5 & 57.9 \\
\hline Aristida adscencionis & 4.7 & 6.5 & 39.5 & 40.6 & 2.8 & 58.1 & Bouteloua sp. & 3.5 & 4.9 & 43.8 & 79.9 & 1.3 & 54.8 \\
\hline Bouteloua aristidoides & 6.7 & 5.0 & 42.4 & 75.6 & 1.3 & 55.9 & Bouteloua aristidoides & 5.3 & 4.7 & 42.4 & 79.0 & 1.2 & 55.9 \\
\hline Eucnide cordata & 4.6 & 8.7 & 35.7 & 36.2 & 3.6 & 61.1 & Eucnide cordata & 3.9 & 7.7 & 38.7 & 46.8 & 2.6 & 58.8 \\
\hline Hibiscus denudatus & 4.8 & 10.7 & 39.0 & 45.7 & 1.9 & 58.6 & Hibiscus denudatus & 4.8 & 8.4 & 40.7 & 55.6 & 1.9 & 57.2 \\
\hline Janusia californica & 6.9 & 9.1 & 36.9 & 47.0 & 2.6 & 60.2 & Senna confinis & 6.5 & 4.5 & 46.6 & 76.5 & 1.7 & 52.6 \\
\hline Merremia aurea & 7.0 & 9.5 & 37.9 & 48.0 & 2.4 & 59.4 & & & & & & & \\
\hline Senna confinis & 8.8 & 5.9 & 46.0 & 71.4 & 2.1 & 53.1 & & & & & & & \\
\hline \multicolumn{14}{|c|}{ Arbustos } \\
\hline Baccharis salicifolia & 4.6 & 6.2 & 35.7 & 58.6 & 4.4 & 61.1 & Baccharis salicifolia & 2.9 & 4.8 & 42.0 & 71.3 & 2.4 & 56.2 \\
\hline
\end{tabular}




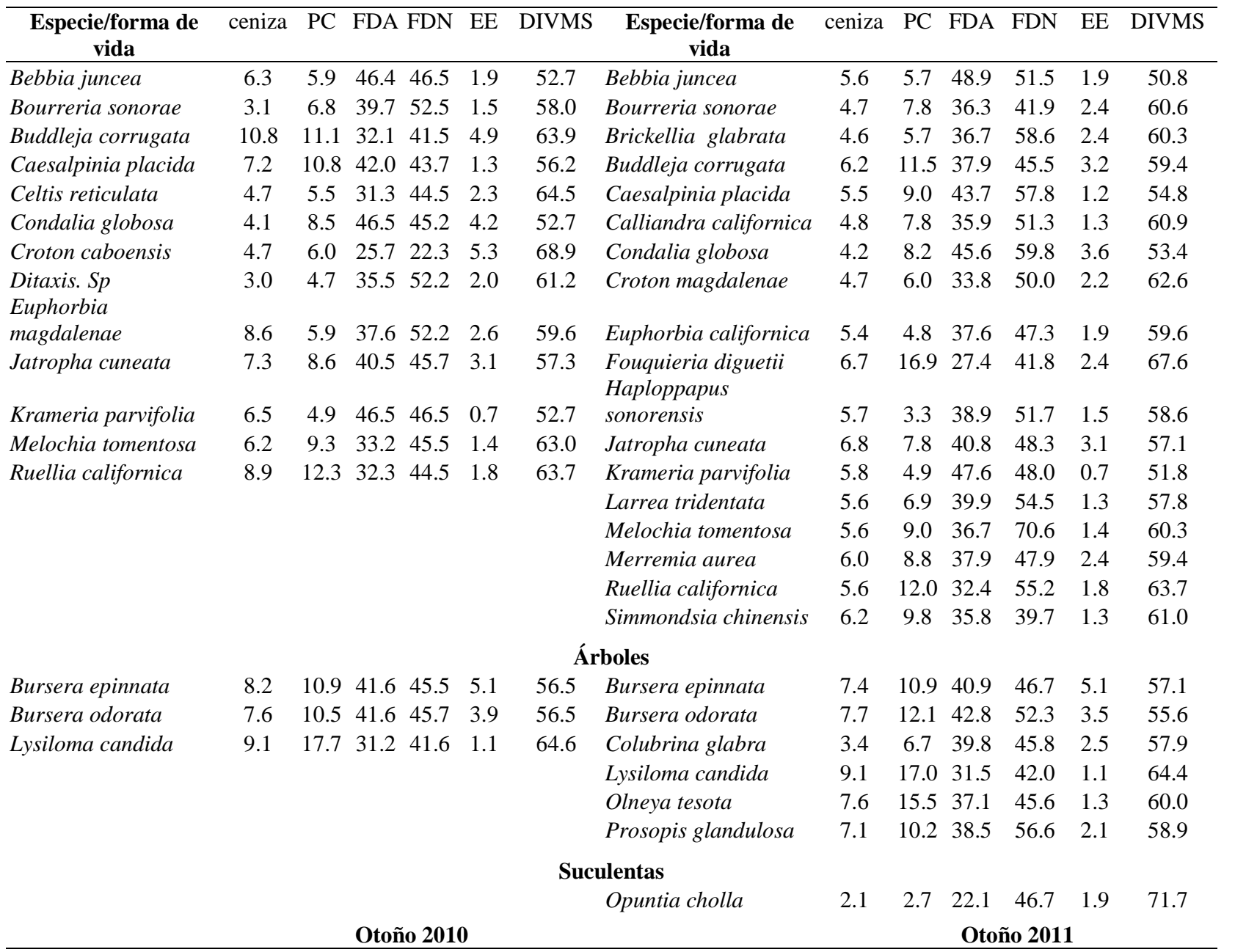

\begin{tabular}{|c|c|c|c|c|c|c|c|c|c|c|c|c|c|}
\hline \multicolumn{14}{|c|}{ Herbáceas } \\
\hline Aristida adscencionis & 4.0 & 5.6 & 45.8 & 45.7 & 2.9 & 53.2 & Aristida adscencionis & 3.6 & 5.7 & 39.9 & 79.7 & 1.9 & 57.8 \\
\hline Atriplex barclayana & 5.6 & 7.2 & 39.9 & 54.7 & 2.5 & 57.8 & Atriplex barclayana & 3.9 & 7.6 & 42.2 & 55.7 & 2.2 & 56.0 \\
\hline $\begin{array}{l}\text { Bouteloua aristidoides } \\
\text { Heteropogon }\end{array}$ & 7.7 & 2.5 & 39.8 & 75.8 & 1.2 & 57.9 & $\begin{array}{l}\text { Bouteloua aristidoides } \\
\text { Heteropogon }\end{array}$ & 5.6 & 4.0 & 41.0 & 79.2 & 1.1 & 57.0 \\
\hline contortus & 5.9 & 4.9 & 40.8 & 75.5 & 1.1 & 57.1 & contortus & 3.5 & 4.3 & 42.6 & 78.6 & 1.1 & 55.7 \\
\hline $\begin{array}{l}\text { Hibiscus denudatus } \\
\text { Hofmeisteria }\end{array}$ & 5.6 & 8.0 & 39.5 & 53.4 & 2.3 & 58.2 & $\begin{array}{l}\text { Hibiscus denudatus } \\
\text { Hofmeisteria }\end{array}$ & 4.4 & 7.9 & 40.7 & 56.3 & 2.3 & 57.2 \\
\hline fasciculata & 4.3 & 7.8 & 40.9 & 44.9 & 4.0 & 57.0 & fasciculata & 2.4 & 6.7 & 40.4 & 44.1 & 1.6 & 57.5 \\
\hline Janusia californica & 6.4 & 9.0 & 36.2 & 46.4 & 2.4 & 60.7 & Senna confinis & 6.5 & 4.3 & 46.9 & 77.0 & 1.4 & 52.4 \\
\hline \multicolumn{14}{|c|}{ Arbustos } \\
\hline Bourreria sonorae & 3.0 & 5.7 & 40.0 & 53.2 & 1.7 & 57.8 & Baccharis salicifolia & 2.4 & 4.5 & 45.7 & 72.5 & 2.2 & 53.3 \\
\hline Buddleja corrugata & 10.9 & 9.1 & 42.2 & 48.4 & 2.8 & 56.0 & Bebbia juncea & 5.6 & 5.9 & 49.2 & 51.9 & 1.6 & 50.5 \\
\hline Caesalpinia placida & 6.9 & 9.7 & 45.5 & 44.9 & 2.6 & 53.5 & Brickellia glabrata & 4.7 & 6.0 & 42.4 & 57.5 & 2.5 & 55.9 \\
\hline Celtis reticulata & 4.5 & 6.7 & 30.8 & 45.7 & 2.5 & 64.9 & Buddleja corrugata & 6.0 & 10.0 & 39.7 & 46.9 & 2.6 & 58.0 \\
\hline Condalia globosa & 3.7 & 9.2 & 45.9 & 56.1 & 4.1 & 53.1 & Caelsalpinia placida & 5.5 & 9.0 & 43.7 & 56.4 & 1.2 & 54.9 \\
\hline Ditaxis. $S p$ & 3.2 & 4.0 & 36.2 & 56.5 & 2.1 & 60.7 & Calliandra californica & 4.0 & 5.8 & 35.8 & 51.5 & 1.4 & 61.0 \\
\hline Krameria parvifolia & 6.6 & 5.8 & 50.2 & 46.3 & 0.7 & 49.8 & Condalia globosa & 4.0 & 8.1 & 45.9 & 60.0 & 3.1 & 53.1 \\
\hline Melochia tomentosa & 6.4 & 9.8 & 32.3 & 45.0 & 1.8 & 63.8 & $\begin{array}{l}\text { Fouquieria diguetii } \\
\text { Haploppapus }\end{array}$ & 5.9 & 17.1 & 27.2 & 41.5 & 2.1 & 67.7 \\
\hline Ruellia californica & 9.8 & 19.8 & 32.3 & 45.2 & 1.8 & 63.7 & sonorensis & 5.7 & 2.9 & 39.0 & 52.2 & 1.6 & 58.5 \\
\hline Simmondsia chinensis & 6.0 & 9.7 & 32.5 & 38.9 & 2.6 & 63.6 & Jatropha cuneata & 6.7 & 7.9 & 41.7 & 48.7 & 3.1 & 56.5 \\
\hline Viscainoa geniculata & 7.3 & 12.3 & 35.1 & 26.9 & 5.2 & 61.6 & Krameria parvifolia & 5.7 & 4.8 & 49.3 & 48.8 & 0.7 & 50.5 \\
\hline
\end{tabular}




\begin{tabular}{|c|c|c|c|c|c|c|c|c|c|c|c|c|c|}
\hline $\begin{array}{c}\text { Especie/forma de } \\
\text { vida }\end{array}$ & ceniza & PC & FDA & FDN & $\mathrm{EE}$ & DIVMS & $\begin{array}{c}\text { Especie/forma de } \\
\text { vida }\end{array}$ & ceniza & PC & FDA & FDN & $\mathrm{EE}$ & DIVMS \\
\hline \multirow[t]{5}{*}{ Xilothamnia diffusa } & 7.0 & 7.6 & 35.9 & 46.4 & 2.4 & 61.0 & Larrea tridentata & 4.8 & 6.5 & 41.4 & 54.7 & 1.3 & 56.7 \\
\hline & & & & & & & Melochia tomentosa & 5.4 & 8.9 & 36.2 & 70.5 & 1.2 & 60.7 \\
\hline & & & & & & & Ruellia californica & 5.6 & 10.7 & 32.3 & 56.8 & 1.8 & 63.7 \\
\hline & & & & & & & Simmondsia chinensis & 6.0 & 9.7 & 35.1 & 39.2 & 1.3 & 61.5 \\
\hline & & & & & & & Vizcainoa geniculata & 6.9 & 9.8 & 31.5 & 40.5 & 4.0 & 64.4 \\
\hline \multicolumn{14}{|c|}{ Árboles } \\
\hline Bursera epinnata & 8.0 & 11.0 & 41.1 & 45.3 & 4.2 & 56.9 & Bursera epinnata & 6.9 & 11.0 & 41.2 & 46.7 & 4.8 & 56.8 \\
\hline \multirow[t]{5}{*}{ Bursera odorata } & 7.7 & 11.5 & 41.1 & 45.7 & 4.3 & 56.9 & Bursera odorata & 7.3 & 12.0 & 42.8 & 52.8 & 3.2 & 55.6 \\
\hline & & & & & & & Colubrina glabra & 3.3 & 5.8 & 40.9 & 46.0 & 3.1 & 57.0 \\
\hline & & & & & & & Olneya tesota & 7.5 & 17.9 & 37.9 & 46.7 & 1.6 & 59.4 \\
\hline & & & & & & & Prosopis glandulosa & 6.5 & 10.6 & 38.7 & 56.7 & 1.9 & 58.8 \\
\hline & \multicolumn{7}{|c|}{ Invierno 2010} & \multicolumn{6}{|c|}{ Invierno 2011} \\
\hline \multicolumn{14}{|c|}{ Herbáceas } \\
\hline Abutilon sp. & 2.2 & 6.6 & 40.5 & 48.3 & 1.9 & 57.3 & Aristida adscencionis & 3.8 & 6.2 & 40.3 & 80.1 & 2.0 & 57.5 \\
\hline Aristida adscencionis & 4.1 & 6.2 & 45.6 & 47.1 & 3.1 & 53.4 & Atriplex barclayana & 3.7 & 7.1 & 42.5 & 56.0 & 2.0 & 55.8 \\
\hline Atriplex barclayana & 5.9 & 8.2 & 41.6 & 48.3 & 4.4 & 56.5 & Bouteloua aristidoides & 5.4 & 3.6 & 46.7 & 78.7 & 1.2 & 52.5 \\
\hline $\begin{array}{l}\text { Bouteloua aristidoides } \\
\text { Heteropogon }\end{array}$ & 5.3 & 3.4 & 46.7 & 78.4 & 1.8 & 52.5 & $\begin{array}{l}\text { Bouteloua sp. } \\
\text { Heteropogon }\end{array}$ & 2.6 & 4.0 & 42.2 & 80.1 & 1.1 & 56.0 \\
\hline contortus & 5.3 & 3.9 & 46.7 & 77.8 & 1.9 & 52.5 & contortus & 4.1 & 3.9 & 44.2 & 78.2 & 1.9 & 54.5 \\
\hline Hibiscus denudatus & 5.8 & 8.2 & 40.7 & 48.2 & 4.2 & 57.2 & Hibiscus denudatus & 4.7 & 7.9 & 41.2 & 56.0 & 4.2 & 56.8 \\
\hline Janusia californica & 6.1 & 8.9 & 38.0 & 47.6 & 3.6 & 59.3 & & & & & & & \\
\hline Sida. Sp & 1.9 & 4.9 & 46.3 & 50.9 & 2.6 & 52.8 & & & & & & & \\
\hline \multicolumn{14}{|c|}{ Arbustos } \\
\hline Aeschynomene nivea & 6.9 & 11.1 & 40.3 & 51.7 & 2.5 & 57.5 & Aeschinomene nivea & 5.0 & 9.3 & 40.9 & 56.2 & 2.2 & 57.0 \\
\hline Baccharis salicifolia & 4.3 & 4.9 & 41.0 & 58.0 & 4.4 & 57.0 & Baccharis salicifolia & 2.2 & 3.6 & 45.9 & 72.6 & 2.0 & 53.1 \\
\hline Bourreria sonorae & 2.8 & 5.5 & 40.3 & 54.1 & 1.8 & 57.5 & Bebbia juncea & 5.4 & 5.4 & 49.4 & 52.1 & 2.4 & 50.4 \\
\hline Buddleja corrugata & 11.3 & 14.3 & 29.8 & 38.7 & 3.5 & 65.7 & Bourreria sonorae & 4.0 & 7.1 & 36.2 & 41.9 & 2.7 & 60.7 \\
\hline Caesalpinia placida & 7.0 & 10.1 & 47.4 & 45.0 & 2.8 & 52.0 & Brickellia glabrata & 4.6 & 4.9 & 40.9 & 57.1 & 2.9 & 57.0 \\
\hline Celtis reticulata & 6.9 & 6.8 & 31.4 & 45.3 & 2.7 & 64.4 & Buddleja corrugata & 5.8 & 12.7 & 39.9 & 46.9 & 2.1 & 57.8 \\
\hline Condalia globosa & 4.2 & 8.0 & 41.3 & 33.5 & 4.0 & 56.7 & Caelsalpinia placida & 4.8 & 8.7 & 45.2 & 56.9 & 1.1 & 53.7 \\
\hline Croton caboensis & 6.0 & 7.1 & 27.5 & 22.6 & 5.7 & 67.5 & Calliandra californica & 4.0 & 5.4 & 35.9 & 52.0 & 1.7 & 60.9 \\
\hline Ditaxis. Sp & 4.1 & 3.7 & 37.5 & 55.3 & 2.4 & 59.7 & Croton magdalenae & 5.2 & 5.8 & 31.5 & 51.2 & 2.0 & 64.4 \\
\hline \multicolumn{14}{|l|}{ Euphorbia } \\
\hline magdalenae & 7.0 & 7.3 & 36.4 & 45.6 & 5.1 & 60.5 & Euphorbia californica & 4.9 & 5.0 & 38.6 & 47.2 & 1.7 & 58.9 \\
\hline Krameria parvifolia & 6.2 & 4.7 & 49.4 & 47.1 & 0.9 & 50.4 & $\begin{array}{l}\text { Fouquieria diguetii } \\
\text { Haploppapus }\end{array}$ & 5.6 & 18.0 & 28.0 & 41.9 & 2.1 & 67.1 \\
\hline Lycium brevipes & 6.9 & 10.1 & 40.0 & 51.3 & 2.7 & 57.8 & sonorensis & 5.2 & 2.7 & 40.1 & 52.5 & 1.8 & 57.6 \\
\hline Melochia tomentosa & 6.1 & 8.0 & 36.6 & 51.2 & 1.8 & 60.4 & Jatropha cuneata & 6.7 & 7.9 & 41.4 & 48.9 & 3.1 & 56.6 \\
\hline Merremia aurea & 5.9 & 8.9 & 39.0 & 48.5 & 4.3 & 58.5 & Krameria parvifolia & 4.4 & 4.6 & 49.9 & 48.7 & 0.7 & 50.1 \\
\hline Ruellia californica & 10.3 & 16.1 & 28.4 & 44.6 & 1.4 & 66.8 & Larrea tridentata & 4.3 & 6.3 & 39.3 & 55.2 & 1.2 & 58.3 \\
\hline Simmondsia chinensis & 6.6 & 9.1 & 33.1 & 40.1 & 2.9 & 63.1 & Lycium brevipes & 6.2 & 10.7 & 42.5 & 55.4 & 1.1 & 55.8 \\
\hline \multirow[t]{6}{*}{ Viscainoa geniculata } & 8.2 & 13.5 & 21.9 & 25.9 & 3.5 & 71.9 & Melochia tomentosa & 5.0 & 8.3 & 35.9 & 71.1 & 1.1 & 60.9 \\
\hline & & & & & & & Merremia aurea & 5.9 & 7.9 & 39.0 & 48.4 & 4.3 & 58.5 \\
\hline & & & & & & & Ruellia caifornica & 6.8 & 10.1 & 29.8 & 57.4 & 1.7 & 65.7 \\
\hline & & & & & & & Simmondsia chinensis & 6.6 & 9.7 & 33.8 & 40.8 & 1.1 & 62.6 \\
\hline & & & & & & & Vizcainoa geniculata & 7.1 & 9.9 & 31.5 & 40.7 & 3.5 & 64.4 \\
\hline & & & & & & & boles & & & & & & \\
\hline \multicolumn{14}{|c|}{56.9 Colubrina glabra } \\
\hline \multirow[t]{5}{*}{ Lysiloma candida } & 9.2 & 14.8 & 45.6 & 42.7 & 3.1 & 53.4 & Bursera epinnata & 6.8 & 10.9 & 41.2 & 47.3 & 4.3 & 56.7 \\
\hline & & & & & & & Lysiloma candida & 9.2 & 15.2 & 35.6 & 42.0 & 1.2 & 61.2 \\
\hline & & & & & & & Olneya tesota & 7.0 & 14.9 & 41.8 & 46.9 & 1.9 & 56.3 \\
\hline & & & & & & Suc & $\begin{array}{l}\text { Prosopis glandulosa } \\
\text { ulentas }\end{array}$ & 7.1 & 9.9 & 40.3 & 57.1 & 1.7 & 57.5 \\
\hline & & & & & & & Opuntia cholla & 2.6 & 2.8 & 22.2 & 47.2 & 1.9 & 71.6 \\
\hline
\end{tabular}

